\title{
K-means clustering for classifying unlabelled MRI data
}

\author{
* Gobert N. Lee and Hiroshi Fujita \\ Department of Intelligent Image Information, Division of Regeneration and Advanced Medical \\ Sciences, Graduate School of Medicine, Gifu University, Gifu, 501-1194, Japan \\ *E-mail: gobert@fjt.info.gifu-u.ac.jp
}

\begin{abstract}
Texture analysis of the liver for the diagnosis of cirrhosis is usually region-of-interest (ROI) based. Integrity of the label of ROI data may be a problem due to sampling. This paper investigates the use of $K$ means clustering, an unsupervised classifier which does not depend on the label of the data, for classification. Moreover, a procedure for generating a ROC curve for $k$-means clustering is also described in this paper. Using a MRI database of 44 patients with 16 cirrhotic and 28 non-cirrhotic liver cases, $k$-means clustering achieves an area under the ROC curve (AUC) index of 0.704. This is comparable to the performance of a linear discriminant analysis (LDA) and an artificial neural network (ANN) with the former attains a resubstitution and an average leave-onecase-out AUC of 0.781 and 0.779 , respectively, and the latter attains a testing AUC of 0.801 .
\end{abstract}

\section{Introduction}

Cirrhosis of the liver is one of the leading causes of death by disease, killing more than 20,000 people in the United States each year. Cirrhosis of the liver is characterized by the presence of widespread fibrosis and regenerative nodules in the liver. The fibrosis and nodules formation causes distortion of the normal liver architecture, resulting in characteristic texture patterns.

Liver biopsy has been, and still is, the gold standard for the diagnosis of cirrhosis. However, advances in imaging technology in recent years results in that hepatic magnetic resonance imaging (MRI) is becoming a very useful imaging modality in examining the liver non-invasively (Figure 1). In interpreting hepatic MRI images, radiologists look for manifestations such as irregularity of the liver boundary, enlargement in the liver, and the presence of fibrosis and regenerative nodules. Interpretation of hepatic texture patterns, however, is proved to be difficult for human observers. This calls for the interest in machine analysis of hepatic texture patterns.

Texture analysis for computer-aided diagnosis (CAD) in medical images has been studied in many disciplines including the diagnosis of breast cancer in mammograms [1-5], lung nodules in chest radiographs [6-8], osteoporosis in bone x-ray images [9-11], and abnormalities in kidney and liver [12-14]. Analysis is typically based on regions-of-interest (ROIs). A classifier is then subsequently used to classify the data (feature vectors) into a number of pre-defined classes. Linear discriminant analysis (LDA), artificial neural network (ANN) and support vector machine (SVM) are among the popular choices of classifiers. The above named classifiers are in the category of supervised classifier which employs a training data set (with known class labels) for training the classification rule.

Problem in diagnosing abnormalities using a scheme involving ROIs and supervised classifier is that a sampled ROI may or may not contain the features that typically represent the disease stage of the organ/structure from which the ROI originated. Yet, disregard of its disposition, the ROI will be given the same label as any other ROI from that organ/structure. If a supervised classifier is subsequently used, the uncharacteristic ROI will constitute an outlier and will have an influence in the training of the classifier.

In this paper, classification of livers as cirrhotic or non-cirrhotic based on texture features measured on ROIs is studied with the use of a different type of classifier, the unsupervised classifier. An unsupervised classifier differs from a supervised classifier in two ways: (1) it works with unlabeled data, and (2) training of a classification rule with the use of a training data set is not required. K-means clustering is a simple and popular unsupervised classifier and is chosen for this study. The ROI texture feature data employed in this study is a subset of the data set reported in Kato et al. [15].

In addition, a method for generating an ROC curve in depicting the classification performance of k-means clustering is also described in this paper. Receiver 
operating characteristic (ROC) analysis [16] is widely used for evaluating performance of classification/CAD schemes, however, schemes employing k-means clustering typically attain only a single measure of sensitivity and specificity. In order to enable full comparisons between classification schemes employing k-means clustering and those employing other classifiers, an ROC curve generation method for $\mathrm{k}$-means clustering is developed.

Finally, with the use of the above ROC curve generating method, the classification results using kmeans clustering are compared with the classification results using a classical LDA and an ANN classifiers, with the former computed in this study and the latter obtained from Kato et. al. [15].

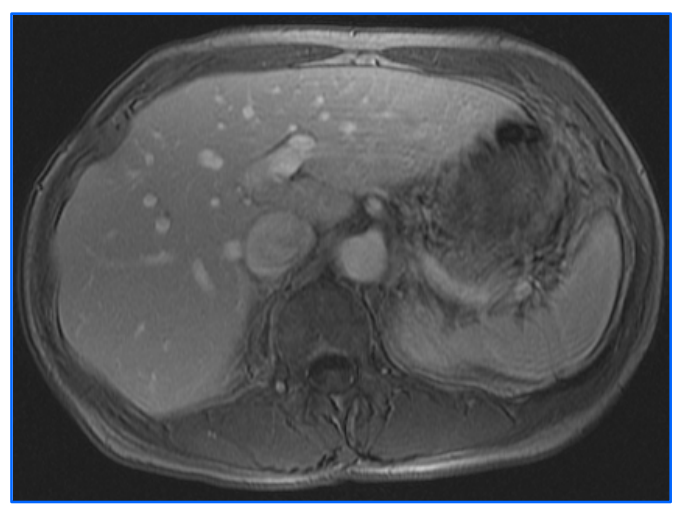

Figure 1. An example of hepatic MRI image.

\section{Method and Materials}

\subsection{MRI Data}

MRI data of a subset of 44 patient cases (16 cirrhotic; 28 non-cirrhotic) reported in a study by Kato et al. [15] was employed in this paper. Details of the MRI data are described in the following.

The above 44 patients were identified from the archive of Gifu University Hospital, Japan in a retrospective study. All the 44 patients had partial hepatectomy for malignant hepatic lesions in the period between February 2000 and January 2002 with preoperative evaluations of hepatic tumors using gadolinium-enhanced MRI performed within 2 weeks prior to surgery. Diagnoses among the 44 patients were hepatocellular carcinoma in cirrhosis, hepatocellular carcinoma in chronic viral hepatitis type C or type B, cholangioccllular carcinoma and liver metastasis. Surgical specimens were retrospectively examined by a pathologist who was blinded to patient histories and radiology and surgery reports. The degree of hepatic fibrosis (Figure 2) in non-tumorous liver parenchyma was evaluated using the 5-point Desmet scale [17] with F0 indicates no fibrosis; F1, mild fibrosis; F2, moderate fibrosis; F3, severe fibrosis; and F4, cirrhosis. For the evaluation of a two-class CAD scheme employed in Kato et al. [15], livers with pathologic grades of F3 and F4 were taken as true positives (cirrhotic livers) and livers with pathologic grades F0-F2 were taken as true negatives (noncirrhotic livers), resulting in a total of 16 true positives and 28 true negatives.

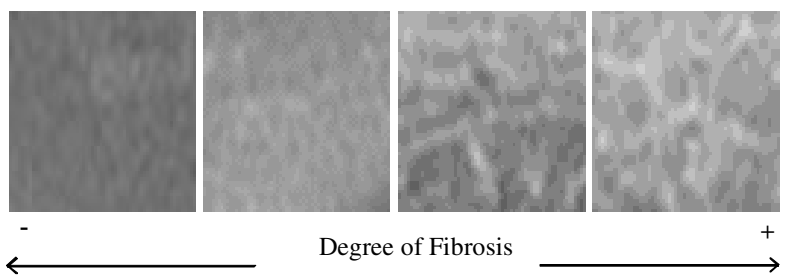

Figure 2. Texture patterns of different degree of fibrosis ranging from normal (left) to cirrhosis (right).

The preoperative hepatic MRI images were acquired using a 1.5T MR scanner (Signa Horizon; GE Medical Systems, Milwaukee, Wis.). MRI images of several sequences were obtained including T1- and T2-weighted MRI as well as gadolinium-enhanced precontrast, hepatic arterial, portal venous and equilibrium phases MRI. All MRI images were acquired in the axial plane. The depth resolution of the images was 16 bits (65536 gray scale levels) and the size of the axial images was $512 \times 512$ pixels with a section thickness of $8-10 \mathrm{~mm}$ and a 2- to 3-mm intersection gap. Depending on the size of the coverage, a MRI sequence of a patient typically consisted of a stack of 18-22 (axial) slice images.

Only the gadolinium-enhanced equilibrium phase images were of interest in this study. This is due to the findings in Kato et al. [15] that performance of the 7 texture features extracted from the gadoliniumenhanced equilibrium phase image is superior to that extracted from other MRI sequence or combination of sequences. From the equilibrium phase images of each patient, 10 ROIs were selected by a radiologist who was blinded to the patient information and the pathologic diagnosis. The ROIs were $32 \times 32$ pixels in size and were placed in the liver parenchyma. Care was taken to avoid large blood vessels, focal hepatic lesions and prominent hepatic artifacts. The locations of the ROIs were planned to cover the liver strategically with eight in the right liver lobe (typically, two in each of 
Couinaud's liver segments V, VI, VII and VIII (Figure 3 )) and two in the left lobe (typically, one in each of Couinaud's liver segments II and III).

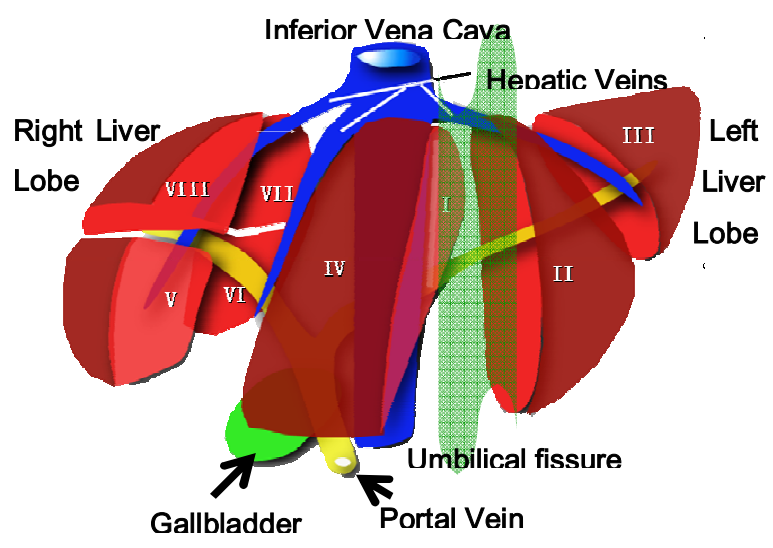

Figure 3. Couinaud's liver segments. The Couinaud classification divides the liver into eight independent segments, each of which has its own vascular inflow, outflow and biliary drainage. Resection of each segment can be performed without damaging the remaining segments.

Furthermore, 7 numeric texture features were measured on each of the ROIs in Kato et al [15]. The seven texture features were contrast, angular second moment, entropy, mean and inverse difference moment derived from co-occurrence matrix [18] as well as the mean and standard deviation derived from the image intensity histogram. Each feature was then averaged over the 10 ROIs. The averaged feature vectors of individual patient case formed the basis of the MRI data employed in this study.

\subsection{K-means clustering}

K-means clustering is one of the simplest unsupervised classification techniques. It is also one of the most popular unsupervised learning algorithms due to its simplicity. As mentioned before, the main differences between a supervised classifier such as the LDA or an ANN, and an unsupervised classifier is that a training data set with known class labels is required for the former to train the classification rule, whereas such a training data set and the knowledge of the class labels in the data set are not required for the latter. In addition, unsupervised clustering techniques are able to discover clusters inherited in the data.
2.1.1. The algorithm. The k-means clustering algorithm partitions a given data set into $k$ mutually exclusive clusters such that the sum of the distances between data and the corresponding cluster centroid is minimized. The above distance measure between two data points is taken as a measure of similarity. A number of distance measures can be used depending on the data. The Minkowski distance, the Euclidean distance and the Mahalanobis distance are some typical examples of distance measures. In this study, the standard Euclidean distance was used as the distance measure.

Mathematically, given a set of data vectors $X=\left[x_{1}\right.$, $\left.\ldots, x_{n}\right]$ where $n$ is the number of observations, the $\mathrm{k}-$ means clustering algorithm groups the data into $k$ clusters with the aim at minimizing an objective function, a squared error function. As the Euclidean distance was adopted as the distance measure in this study, the objective function $J$ is then explicitly given as

$$
J=\sum_{j=1}^{k} \sum_{i \in c_{j}}\left\|x_{i}-\mu_{j}\right\|^{2},
$$

where $c_{j}$ is the $j$-th cluster and $\mu_{j}$, is the centroid of the cluster $c_{j}$. Therefore, the k-means clustering algorithm is an iterative algorithm that finds a suitable partition which minimizes the sum squared error. The algorithm begins with the initialization of $k$ cluster centroids. Different approaches in initialization have been suggested. A simple method is to initialize the problem by randomly select $k$ data points from the given data. The remaining data points are classified into the $\mathrm{k}$ clusters by distance. The centroids are then updated by computing the centroids in the $k$ clusters.

To summarize, there are different categories of clustering techniques including partitioning, hierarchical, density-based and grid-based clustering. The k-means clustering algorithm is a clustering technique that falls into the category of partitioning. The algorithm finds a partition in which data points within a cluster are close to each other and data points in different clusters are far from each other as measured by similarity. As in other optimization problems with a random component, the results of $k$ means clustering are initialization dependent. This is usually dealt with by running the algorithms several times, each with a different initialization. The best solution from the multiple runs is then taken as the final solution.

2.1.2. Determining the number of clusters. The $k$ means clustering algorithm requires the knowledge of $k$, the number of clusters. Unfortunately, this is usually 
not known a prior. Furthermore, real data are often corrupted with noise and the clusters in real data sets are often not well separated. As a result, the data set can be interpreted with several possible numbers of clusters. This means determining the number of clusters is an ill-posed problem. Nevertheless, a number of approaches have been suggested in finding the number of clusters. Here, the number of clusters is determined by assessing the 'quality' of the clustering results over a range of $k$.

In this study, the silhouette index was employed as a cluster validity index. A silhouette index was defined on each and every data point (feature vector) in the feature space. The silhouette index of the $i$-th data point is given by

$$
S_{i}=\frac{b_{i}-a_{i}}{\max \left(a_{i}, b_{i}\right)},
$$

where $a_{i}$ is the average distance of the $i$-th point to other points in the same cluster and $b_{i}$ is the average distance of the $i$-th point to points in its nearest neighbour cluster. The silhouette index indicates how well a data point is clustered. In particular, the silhouette index $S_{i}$ is a normalized difference measure of the average distance of the $i$-th point to all other points in the same cluster and that of the $i$-th point to all other points in the closest cluster with distance being the Euclidean distance. The value of the silhouette index $S_{i}$ ranges from +1 to -1 . A value of unity indicates that the point is very distant from its neighbor clusters while a value of -1 indicates that the point is closer to points in its nearest neighbor cluster than to those in its own cluster.

An average silhouette is obtained by averaging the silhouette values over the number of data points. The average silhouette

$$
S(k)=\frac{1}{N} \sum_{i=1 \ldots N} S_{i}
$$

is an index measuring the overall 'quality' of the partition where $S_{i}$ is the silhouette index defined in Equation 2 and $N$ is the number of observations. Similar to the point silhouette index, a high value in the average silhouette indicates the 'quality' of the partition is good. Furthermore, the number of clusters, $\mathrm{k}$, is a parameter of the average silhouette. The number of clusters can be determined by maximizing $S(k)$ over a range of $k$.

\subsection{ROC curve for $k$-means clustering}

In evaluating a classification/CAD scheme, ROC analysis [16] is widely used. An ROC curve is a plot of the true positive fraction (tpf) verses the false positive fraction (fpf). It depicts the trade-off between the sensitivity and the specificity. For multivariate observations, data can be transformed into univariates using, for example, a linear discriminant function. The probability density functions of the transformed univariates can then be easily computed. If a linear discriminant function is used in the transformation, the transformed value is often referred to as the discriminant score.

From the perspective of ROC curve generation, the probability density functions of the transformed univariates are the underlying curves from which the ROC curve is generated. Furthermore, the decisionvariable in generating the ROC curve is defined on the axis of the transformed univariates (Figure 4).

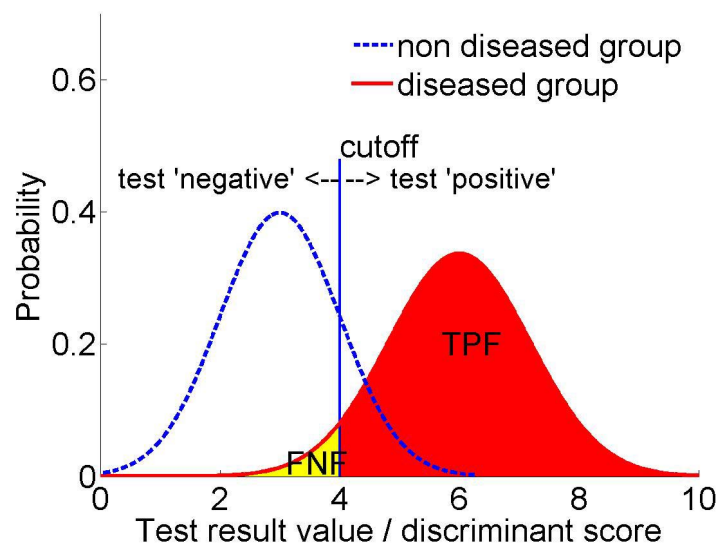

Figure 4 . The underlying probability density functions that generate an ROC curve.

For classifiers such as the LDA and ANN, output of the classifier is a univariate, hence, ROC curve construction is straightforward. However, it should be noted that in the above two classifiers, the support for the LDA output is [-inf, inf] while that for the ANN output is $[0,1]$. Consequently, assumption of a binormal ROC curve would not be suitable for the ANN classifier,

Contrary to LDA and ANN, k-means clustering does not transform the multivariate feature input into a univariate. This makes the construction of an ROC curve non-trivial. In the remaining of this Section, a 
method in generating an ROC curve depicting the kmeans clustering performance is described. The method is a non-parametric method. It takes on any kmeans clustering output without making any assumptions of the distributions or density functions of the clusters. An iterative procedure is employed in order to obtain a number of (tpf, fpf) ROC curve data points. The procedure is described as follows.

1. Apply $k$-means clustering with $k=2$.

2. Compute the fractions $T_{1}$ and $T_{2}$ of cirrhotic livers detected in clusters $\mathrm{C}_{1}$ and $\mathrm{C}_{2}$, respectively.

3. Determine the true positive cluster by comparing $\mathrm{T}_{1}$ and $\mathrm{T}_{2}$, that is,

$$
\left\{\begin{array}{lll}
C_{1}=\text { true positive cluster } & \text { if } & T_{1}>T_{2} \\
C_{2}=\text { true positive cluster } & \text { if } & T_{2}>T_{1}
\end{array} .\right.
$$

4. Compute (tpf, fpf).

5. Relocate a point $x_{i}$ from $\mathrm{C}_{1}$ to $\mathrm{C}_{2}$ such that the change in $J$, as shown in Equation 1, is minimum.

6. Compute (tpf, fpf).

7. Repeat Step 5- 6 until $C_{1}$ becomes an empty cluster.

8. Reinstate the $\mathrm{k}$-means clustering results.

9. Relocate a point $x_{i}$ from $\mathrm{C}_{2}$ to $\mathrm{C}_{1}$ such that the change in $J$, as shown in Equation 1, is minimum.

10. Compute (tpf, fpf).

11. Repeat Step 9 - 10 until $\mathrm{C}_{2}$ becomes an empty cluster.

Once all the (tpf, fpf) data points are obtained, the ROC curve can be generated by simply plotting the true positive fraction verses the false positive fraction.

\section{Results and discussions}

\subsection{Number of clusters}

In determining the number of clusters $k$, a range of values were investigated. For each $k$, the $k$-means clustering algorithm was run 50 times. The best solution with the least sum of distances (Equation 1) was taken as the final solution for that particular $\mathrm{k}$. Finally, the average silhouette index $S(k)$ was used in assessing the cluster validity with different $k$. The average silhouette index with a range of $k$ is shown in Figure 5. It can be seen that $S(k)$ increases monotonically in the range $k=2, \ldots 8$. Consider the small size of the data set, dividing the data into as many as 8 clusters would mean that each cluster would contain a few data. The monotonic increase in $S(k)$ in this scenario was thought to be due to the classes in the data are not well separated. Figure 5 also shows that there are two regions where the increase of $S(k)$ is small. The two regions are from $k=2$ to $k=3$, and from $k=6$ to $k=7$. The small increase in $S(k)$ indicates that the clusters are fairly stable. Due to the small number of samples in this study, the region with a smaller $k$, that is, $k=2$ was taken as the optimal number of clusters.

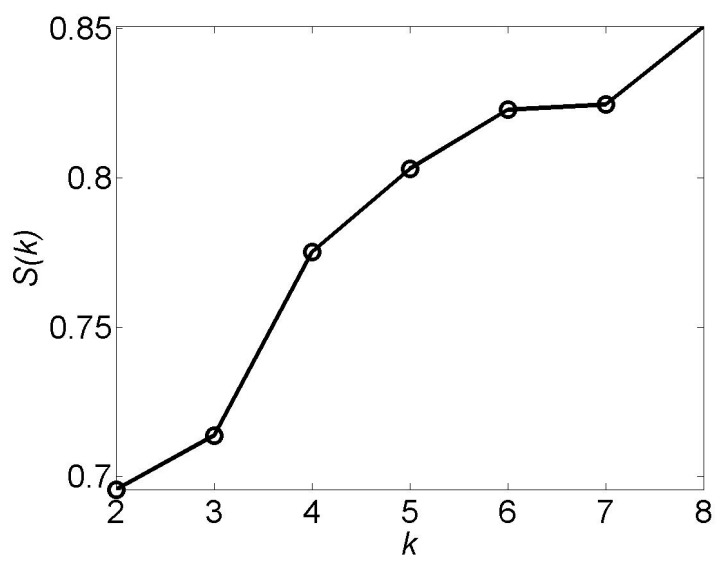

Figure 5. Plot of the average silhouette index $S(k)$ for $k=2,3, \ldots 8$.

\subsection{Classification results}

Using k-means clustering and the proposed ROC curve generating method, the area under the ROC curve (AUC) was found to be 0.704 (Figure 5).

In order to assess how well the unsupervised classifier performed in the classification task, the performance of the k-means clustering was compared to that of the LDA and an ANN using the same MRI feature data. For the LDA classifier, the resubsitition AUC index was found to be 0.781 (Figure 5) and the mean leave-one-case-out AUC index was found to be 0.779 (range $0.760-0.821$ ). For the ANN classifier, the results shown in Kato et al. [15] were used here. Using a separate 8 patient cases (not available to this study) for training the ANN and the same 44 patient cases as in this study for testing, an AUC of 0.801 was claimed in their study. 


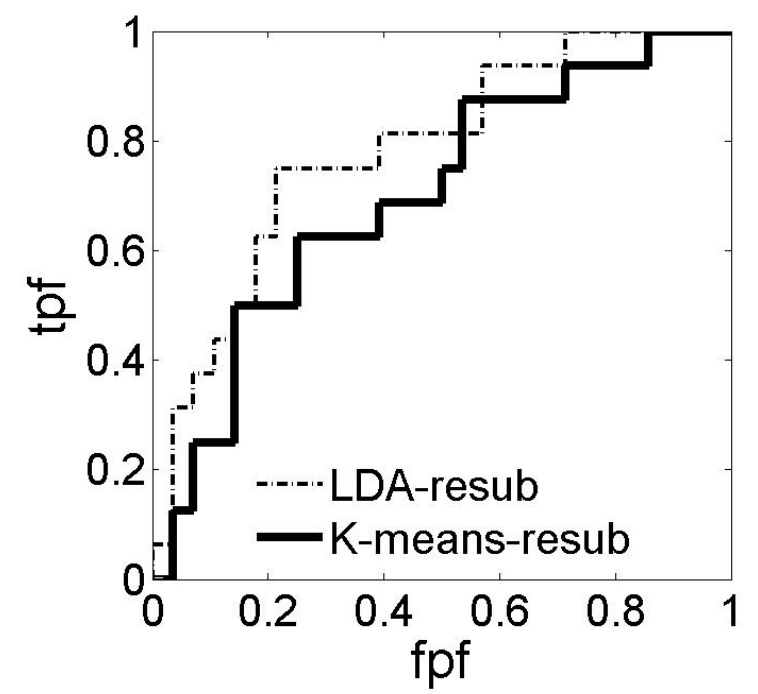

Figure 6. ROC curves obtained using k-means clustering and linear discriminant analysis (LDA). Resubstitution scheme was used in both situations. The area under the ROC curve (AUC) indices were 0.781 for LDA and 0.704 for k-means clustering.

\section{Conclusions}

K-means clustering is an unsupervised classifier. The use of an unsupervised classifier eliminates the dependency of the class labels in the data but may be subjected to loss of information which could affect the goodness of the classifier. The 'goodness' of $k$-means clustering was assessed by comparing classification results with other commonly used classifiers including the LDA and an ANN. It was found that classification results using k-means clustering, the LDA and an ANN were found comparable.

In addition, a procedure for generating a ROC curve in depicting the classification performance of k-means clustering was described. The use of ROC analysis for evaluation is becoming a trend for medical diagnosis/CAD schemes. The proposed ROC generating procedure enable k-means clustering to be evaluated against other classifier on a common ground.

\section{Acknowledgements}

The authors would like to thank Dr. Hiroki Kato, MD, Dr. Masayuki Kanematsu, MD, and Hiroaki Hoshi, MD from the Department of Radiology, Gifu University School of Medicine, Gifu, Japan, for providing the MRI data and discussing their results. The authors would also like to thank the staff and members of the Department of Intelligent Image Information, Gifu University and the Department of Radiology, Gifu University School of Medicine for their helpful technical support and assistance in many areas. This research was supported in part by a grant for the Knowledge Cluster Gifu-Ogaki (KCGO), referred to as the "Robotics Advanced Medical Cluster," from the Ministry of Education, Culture, Sports, Science and Technology, Japan.

\section{References}

[1] S. Sahiner, H.-P. Chan, and N. Petrick et al., "Computerized characterization of masses on mammograms: the rubber band straightening transform and texture analysis", Med. Phy. Vol.25(4), 1998, pp.516-526.

[2] N.R. Mudigonda, R.M. Rangayyan and J.E.L. Desautels, "Gradient and texture analysis for the classification of mammographic masses", IEEE Trans. Med. Imag., Vol.19(10), 2000, pp.1032-1043.

[3] R. Gupta and P.E. Undrill, "The use of texture analysis to delineate suspicious masses in mammography", Phys. Med. Biol. Vol.40, 1995, pp.835-855

[4] H-P. Chan, B. Sahiner, and N. Petrick et. al. "Computerized classification of malignant and benign microcalcifications on mammograms: texture analysis using an artifical neural network", Phys. Med. Biol., Vol. 42, 1997, pp.549-567.

[5] M. Zhang, M.L. Giger, and K. Doi, "Mammographic texture analysis for the detection of spiculated lesions", In: 3rd Int'l Workshop on Digital Mammography (eds. K. Doi, M. L. Giger, R. M. Nishikawa, R. A. Schmidt), Elsevier Science, Amsterdam, pp. 347-350, 1996.

[6] X. Chen, K. Doi, and S. Katsuragawa et al., "Automated selection of regions of interest for quantitative analysis of lung textures in digital chest radiographs", Med. Phys., Vol.20, 1993, pp.975-982.

[7] B. van Ginneken, S. Katsuragawa, and B. ter Harr Romeny B et al., "Automatic detection of abnormalitites in chest radiographs using local texture analysis", IEEE Trans Med Imag., Vol.21(2), 2002, pp.139-149.

[8] E.A. Hoffman, J.M. Reinhardt, and M. Sonka et. al, "Characterization of the interstitial lung diseases via densitybased and texture-based analysis of computed tomography images of lung structure and function", Acad. Radiol. Vol.10(10), 2003, pp.1104-1118.

[9] L. Lan, M.L. Giger, and J. R. Wilkie et al., "Automated selection of region of interest for radiographic texture 
analysis", Proc SPIE Medical Imaging, Vol. 6514, 2007, pp. 651436

[10] M.R. Chinander, M.L. Giger, and R.D. Shah et al., "Investigation of using bone texture analysis on bone densitometry images", Proc SPIE, Vol. 4684, 2002, pp.860863, 2002.

[11] J.R. Wilkie, M.L. Giger, and M.R. Chinander et al., "Comparison of radiographic texture analysis from computed radiography and bone densitometry systems", Med. Phys. Vol.31, 2004, pp.882-891.

[12] G.N. Lee, X. Zhang, and M. Kanematsu et al., "Classification of cirrhotic liver on MR images using texture analysis", Int'l J. CARS, Vol.1, Supp. 1, 2006, pp.379-381.

[13] H. Yoshida, D. Casalino, and B. Keserci et al., "Wavelet packet based texture analysis for differentiation between benign and malignant liver tumors in ultrasound images", Phys. Med. Biol. Vol. 48, 2003, pp.3735-3753.
[14] D.-Y. Kim and J.-W. Park, "Computer-aided detection of kidney tumor on abdominal computed tomography scans", Acta Radiol. Vol.45, 2004, pp.791-795.

[15] H. Kato, M. Kanematsu, and X. Zhang et al., "Computer-aided diagnosis of hepatic fibrosis: preliminary evaluation of MRI texture analysis using the finite difference method and an artificial neural network", AJR, Vol.189, 2007, pp.117-122.

[16] C.E. Metz, "Basic principles of ROC analysis", Seminars in Nuclear Medicine, Vol.8, 1978, pp.283-298.

[17] V.J. Desmet, M. Gerber, and J.H. Hoofnagle et al., "Classification of chronic hepatitis: diagnosis, grading and staging", Hepatology, Vol. 19, 1994, pp.1513-1520.

[18] R.M. Haralick, K. Shanmugam, and I. Dinstein, "Texture features for image classification", IEEE Trans. Sys. Man Cybern. Vol. SMC-3-6, 1973, pp.610-621. 